\title{
Thermodynamic phase retrieval of convective clouds: impact of sensor viewing geometry and vertical distribution of cloud properties
}

\author{
E. Jäkel, J. Walter, and M. Wendisch \\ Leipzig Institute for Meteorology (LIM), University of Leipzig, Leipzig, Germany \\ Correspondence to: E. Jäkel (e.jaekel@uni-leipzig.de)
}

Received: 14 September 2012 - Published in Atmos. Meas. Tech. Discuss.: 22 October 2012

Revised: 8 February 2013 - Accepted: 8 February 2013 - Published: 1 March 2013

\begin{abstract}
The sensitivity of passive remote sensing measurements to retrieve microphysical parameters of convective clouds, in particular their thermodynamic phase, is investigated by three-dimensional (3-D) radiative transfer simulations. The effects of different viewing geometries and vertical distributions of the cloud microphysical properties are investigated. Measurement examples of spectral solar radiance reflected by cloud sides (passive) in the near-infrared (NIR) spectral range are performed together with collocated lidar observations (active). The retrieval method to distinguish the cloud thermodynamic phase (liquid water or ice) exploits different slopes of cloud side reflectivity spectra of water and ice clouds in the NIR. The concurrent depolarization backscattering lidar provides geometry information about the cloud distance and height as well as the depolarization.
\end{abstract}

\section{Introduction}

Clouds are a dominant modulator of the earth's climate (IPCC, 2007). Depending on the cloud properties (top and bottom height, thermodynamic phase, optical thickness, and droplet or particle size), they have the potential to either cool or warm the atmosphere beneath the cloud. Different processes may influence the coagulation (collision and coalescence) and freezing mechanisms inside clouds which determine the precipitation formation, the lifetime and vertical extent of the cloud (Rosenfeld, 2000; Koren et al., 2004; Lohmann and Feichter, 2005; Khain et al., 2008). Rosenfeld and Lensky (1998) found that the relationship between temperature or height and cloud particle effective radius provides significant information on the precipitation-forming processes in convective clouds. To investigate these complex interactions, vertical profile measurements of microphysical (such as thermodynamic phase, cloud particle size and liquid or ice water content) and radiative properties are essential.

Traditionally, vertical profiling of clouds is based mostly on radar and lidar or in situ measurements. Radar and lidar are not always available on aircraft. The sample volume of in situ measurements is restricted to the flight path of the aircraft. Also, characterizing the vertical structure of a cloud is biased by the temporal evolution of the cloud while the aircraft is climbing from cloud base to cloud top. For deep convective clouds strong turbulence complicates in situ probing.

Active remote sensing provides profiles along the lineof-sight, depending on the penetration depth of the radiation source (either coherent monochromatic electromagnetic waves in case of lidar or radio waves for radar). In general, lidar does not penetrate deeply into the cloud (up to an optical depth of 2-4), but it provides detailed information about the optical properties of the cloud edges. Radar may penetrate through a cloud but the quantitative retrieval of cloud optical and microphysical properties is uncertain since the signal is dominated by scattering through large droplets. Lidar measurements of the degree of linear polarization can be used to determine the thermodynamic phase (Scotland et al., 1971; Sassen, 1991). The quantity to describe the degree of linear depolarization is the linear depolarization ratio $\delta$ which is the ratio of the perpendicular and parallel polarized backscattered intensities with respect to the transmitter polarization plane. Non-spherical particles such as ice crystals may rotate 
the oscillation plane of the electric and magnetic field vectors, thus causing significant depolarization. Multiple scattering increases the depolarization ratio $\delta$ with penetration depth in liquid water clouds. Therefore, one must examine the changes of $\delta$ with penetration depth, and thereby can one get information about the thermodynamic phase of the cloud (Hu et al., 2001).

Spaceborne and airborne passive remote sensing methods are based on measuring the reflected solar and emitted terrestrial radiances. Such observations have successfully been applied to retrieve the cloud macro- and microphysicalstructure. The retrieval of vertical profiles from nadir or zenith radiance observations is inherently limited to determine either bulk properties integrated over the entire column (like the optical thickness) or to quantities representative of limited cloud portions depending on cloud thickness (like the thermodynamic phase or droplet size). Several publications have shown studies concerning the derivation of vertical properties of cloud layers from satellite observation. They are based on spectral differences in penetration depths of NIR radiation using the concept of weighting functions (Platnick, 2000; Wang et al., 2009; Zhang et al., 2010). Chang and $\mathrm{Li} \mathrm{(2002)}$ and Chang and $\mathrm{Li}$ (2003) proposed a method for retrieving the vertical profile of effective radius for stratiform clouds by combining the reflectances at three absorbing near-infrared wavelength bands (1.6, 2.1, $3.7 \mu \mathrm{m})$. Recently, Kokhanovsky and Rozanov (2012) presented an approach for shallow warm clouds which uses the optimal estimation method and direct radiative transfer simulations of respective weighting functions. However, vertical information of deep convective clouds cannot be derived from nadir observations applying these methods. Current satellite and aircraft retrieval methods are based mostly on one-dimensional (1-D) radiative transfer simulations which assume that clouds are horizontally homogeneous. While such an approach might be feasible for a cloud-top-viewing instrument, for the proposed cloud-side-scanning geometry the consideration of 3$\mathrm{D}$ effects is mandatory. Zinner et al. (2008) and Martins et al. (2011) presented an airborne cloud scanner that measures spectral radiances reflected from cloud sides which potentially allows for the retrieval of the vertical profile of cloud droplet sizes near cloud edges. Assuming non-precipitating clouds this vertical distribution corresponds to the vertical profile of the whole cloud (Rosenfeld and Lensky, 1998; Freud et al., 2008).

Retrieving the effective radius requires knowledge about the thermodynamic phase, because the optical properties of ice and liquid water clouds deviate significantly. The retrieval of the thermodynamic phase is often based on two methods, one using the different emissivity of ice and liquid water particles in the thermal infrared (TIR: 5-50 $\mu \mathrm{m}$ ) wavelength range (Strabala and Ackerman, 1994; Baum et al., 2000; Turner et al., 2003); the other approach applies cloud reflectivity measurements in the near infrared (NIR: 0.7$2.5 \mu \mathrm{m}$ ), where the refractive indices of the cloud particles of both phases are different (Pilewskie and Twomey, 1987; Knap et al., 2002; Acarreta et al., 2004; Ehrlich et al., 2008). For example, the imaginary part of the refractive index determining the absorption of electromagnetic radiation due to ice is larger than that for liquid water in the wavelength range between 1.5-1.7 $\mu \mathrm{m}$. Therefore, the ratio of cloud reflectances at two wavelengths can be used to determine the cloud thermodynamic phase. For satellite-based data of the Scanning Imaging Absorption Spectrometer for Atmospheric CHartographY (SCIAMACHY) Acarreta et al. (2004) used this ratio-method and defined a cloud phase index which is calculated by fitting the spectral slope of the reflectivity around $1.6 \mu \mathrm{m}$ and its normalization with the reflectivity at $1.64 \mu \mathrm{m}$. Knap et al. (2002) and Ehrlich et al. (2008) applied the ratio-method based on airborne measurements of nadir reflectances while Martins et al. (2011) used reflectivity at $2.10 \mu \mathrm{m}$ and $2.25 \mu \mathrm{m}$ from cloud sides. Ground-based measurements of cloud side reflectivity were performed by Pilewskie and Twomey (1987). They used normalized spectra between 1.5 to $1.7 \mu \mathrm{m}$ to derive the cloud phase.

Compared to previous publications by Martins et al. (2011) and Pilewskie and Twomey (1987) this paper introduces a retrieval method which is systematically investigated with respect to viewing geometry and vertical distribution of cloud properties by 3 -D radiative transfer simulations. The spectral signature between $1.5-1.7 \mu \mathrm{m}$ of the reflected radiation is used to identify the cloud phase. Additionally, collocated lidar data give important information about the viewing geometry. It is studied whether there is a specific threshold that distinguishes between liquid, ice and mixed cloud phase. Section 2 describes the 3-D radiative transfer model (RTM) and the instrumentation used in this work. In Sect. 3 sensitivity studies of the derived cloud phase with respect to cloud microphysics and viewing geometry based on RTM results are presented. Finally, the application of the phase discrimination method is examined by a case study of observations in Sect. 4.

\section{Methods and materials}

\subsection{Modeling}

Radiative transfer simulations are performed with the opensource Monte Carlo Atmospheric Radiative Transfer Simulator (MCARATS), which is a forward-propagating Monte Carlo photon-transport model (Iwabuchi, 2006; Iwabuchi and Kobayashi, 2008). It traces individual photons on their path through the 3-D atmosphere. To improve the computational effort for radiance simulations MCARATS uses several variance reduction techniques as a modified local estimation method or a truncation approximation for highly anisotropic phase functions (Iwabuchi, 2006). Input to the radiative transfer model (RTM) are the optical properties of the atmosphere (e.g., extinction coefficients, single scattering 
albedos, phase functions) and the surfaces albedo. The model domain is divided into grid cells. The user is required to specify 3-D layers for horizontal inhomogeneous distributions of cloud or aerosol particles. Other layers can be defined as horizontally homogeneous, as applied for the optical properties of gaseous constituents. Profiles of atmospheric pressure, temperature, density, and gases are taken from profiles given by Anderson et al. (1986). Molecular (Rayleigh) scattering is calculated from the density profile according to Bodhaine et al. (1999). For gas absorption the LOWTRAN (Low Resolution Transmission Model) parametrization by Pierluissi and Peng (1985), as adapted from SBDART (Santa Barbara DISORT Atmospheric Radiative Transfer) (Ricchiazzi and Gautier, 1998) are used. The optical properties of liquid water and ice clouds with profiles of effective radius $\left(r_{\text {eff }}\right)$ and liquid (ice) water content (LWC, IWC) are specified in 3-D layers. The microphysical properties of liquid water clouds are converted to optical properties by Mie calculations, while for ice clouds the parameterizations by Baum et al. (2005, 2007) are applied. Further input variables are the extraterrestrial solar spectrum from Gueymard (2004) and the solar zenith and azimuth angles $\left(\theta_{0}, \varphi_{0}\right)$ as well as sensor zenith and azimuth angles $\left(\theta_{\mathrm{s}}, \varphi_{\mathrm{s}}\right)$.

\subsection{Instrumentation}

Lidar and spectroradiometer measurements are performed by the LIdar and RAdiation System for cloud profiling (LIRAS). The instrument setup is schematically shown in Fig. 1. Lidar and optical inlet of the spectroradiometer are mounted on a joint angular tracker with an angular resolution of about $1^{\circ}$ to scan cloud sides simultaneously with passive and active sensors. The field of view (FOV) of the radiance optical inlet is about $1^{\circ}$, and thus larger than that of the lidar $\left(0.114^{\circ}\right)$, which results in a different footprint diameter. For example, for a cloud distance of $10 \mathrm{~km}$ the footprints are about $170 \mathrm{~m}$ (radiance inlet) and $20 \mathrm{~m}$ (lidar), respectively.

The spectroradiometer is a ground-based version of the airborne SMART (Spectral Modular Airborne radiation measurement system). It provides measurements of radiances covering almost the entire solar spectral range (Wendisch et al., 2001; Bierwirth et al., 2009; Jäkel et al., 2005; Ehrlich, 2009). The radiation is collected by an entrance optic and transmitted via bifurcated optical fiber to two spectrometers; one for the visible (VIS) spectral range, the other for the NIR. Within the spectrometers a fixed-grating disperses the radiation into spectral components which are detected by a linear photodiode array. The measurement uncertainty of the spectroradiometer includes calibration lamp and transfer calibration uncertainties (between laboratory and field) as well as the wavelength accuracy of the spectrometers. That gives total relative uncertainties in the visible spectral range of $6 \%$ and $9 \%$ for the NIR (Eichler et al., 2009).

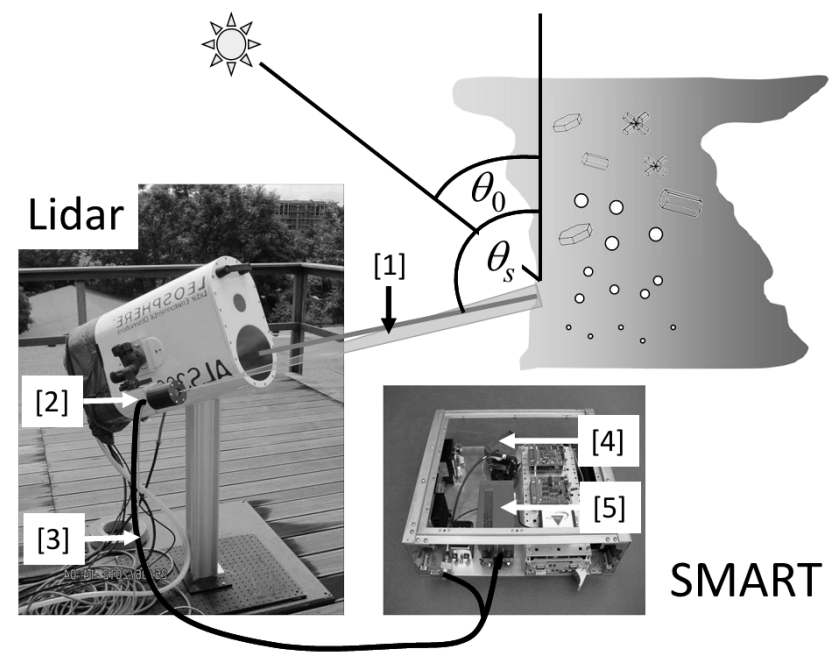

Fig. 1. LIRAS setup consisting of depolarization lidar and SMARTspectroradiometer. (1) Schematic field of view (FOV) of both instruments, (2) radiance optical inlet, (3) optical fiber, (4) NIR spectrometer, and (5) VIS spectrometer. Additionally, the sensor and solar zenith angle $\left(\theta_{\mathrm{s}}, \theta_{0}\right)$ are illustrated.

For this application a phase index $I_{\mathrm{p}}$ is defined from the spectral slope of the radiance at $1550 \mathrm{~nm} I_{1550}$ and $1700 \mathrm{~nm}$ $I_{1700}$, respectively:

$I_{\mathrm{p}}=\frac{I_{1700}-I_{1550}}{I_{1700}}$.

The definition of this index is based on differences in spectral shapes of reflected radiances as presented in Fig. 2 which shows examples of measured NIR radiance spectra of ice and liquid water clouds reflected from cloud sides. The spectral signature of radiance cloud spectra is mainly different within the range between 1.5 and $1.7 \mu \mathrm{m}$. In this case, the slope is positive for ice clouds and negative for liquid water clouds. In Sect. 3 it will be shown by radiative transfer simulation, if this is a general characteristic for variable viewing geometries and cloud properties.

The depolarization backscattering lidar system (ALS300 from Leosphere, France) is primarily used for geometric information as cloud height and cloud distance. In addition, the slope of the depolarization can give an indication on the thermodynamic phase. Table 1 summarizes the technical specifications of LIRAS.

\section{Sensitivity study}

\subsection{Homogeneous cloud}

3-D radiative transfer simulations of reflected radiances on cloud sides are performed for (i) different microphysical parameters (cloud particle size, water content) and (ii) sensor viewing geometries that is described by the solar azimuth and 


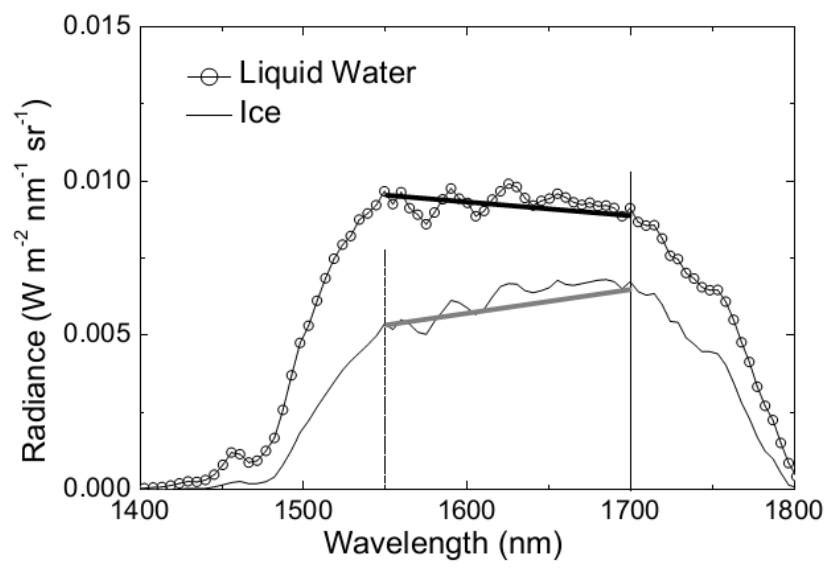

Fig. 2. Measured spectral radiance of cloud edges with ice cloud and liquid water cloud particles.

Table 1. Technical specifications of LIRAS. FWHM: full width at half maximum.

\begin{tabular}{lll}
\hline & $\begin{array}{l}\text { Zeiss } \\
\text { Spectrometer }\end{array}$ & $\begin{array}{l}\text { Lidar } \\
\text { (Leosphere ALS300) }\end{array}$ \\
\hline Wavelength $(\mathrm{nm})$ & $350-2000$ & 355 \\
Spectral Resolution $(\mathrm{nm})$ & FWHM $_{\mathrm{VIS}}=3$ & - \\
& $\mathrm{FWHM}_{\mathrm{NIR}}=16$ & \\
& $\Delta \lambda_{\mathrm{VIS}}=0.8$ & \\
& $\Delta \lambda_{\mathrm{NIR}}=5$ & \\
Accumulation Time (s) & 1 & $10-30$ \\
Field of View $\left(^{\circ}\right)$ & 1 & 0.114 \\
Polarization & - & Vertical and Parallel \\
\hline
\end{tabular}

solar zenith angle $\left(\varphi_{0}, \theta_{0}\right)$ and the sensor azimuth and sensor zenith angle $\left(\varphi_{\mathrm{s}}, \theta_{\mathrm{s}}\right)$. From these calculations at $1.55 \mu \mathrm{m}$ and $1.7 \mu \mathrm{m}$ the phase index $I_{\mathrm{p}}$ was derived using Eq. (1). The model domain has $140 \times 40 \times 139$ grid cells with a horizontal resolution grid cell of $250 \mathrm{~m}$ and a vertical resolution of $200 \mathrm{~m}$ below $22 \mathrm{~km}$ and variable resolution above. A homogeneous cloud $(15 \times 40 \times 16$ grid cells $)$ either consisting of liquid water droplets or of ice crystals is placed into the center of the model domain between 3.8 and $7.0 \mathrm{~km}$ altitude. MCARATS simulates radiances for all $140 \times 40$ grid points at surface altitude.

First, the impact of the microphysical parameters $r_{\mathrm{eff}}$ and LWC/IWC is studied for a fixed viewing geometry $\left(\theta_{\mathrm{s}}=50^{\circ}\right.$, $\theta_{0}=30^{\circ}, \varphi_{\mathrm{s}}=\varphi_{0}=0^{\circ}$ ). For all cases shown in Fig. 3, the phase index of liquid water, regardless of particle size, is lower than zero, whereas ice clouds show a positive phase index. Due to the increase of absorption with increasing particle size, the largest phase index is derived for ice particles with $r_{\text {eff }}=50 \mu \mathrm{m}$. Less absorbing particles (as particles with decreasing size) lead to lower values of $I_{\mathrm{p}}$. Additionally, Fig. 3 illustrates that for all particle sizes the phase index is most variable, more than $20 \%$, for water content values below $0.4 \mathrm{~g} \mathrm{~m}^{-3}$. Above that threshold the variation of $I_{\mathrm{p}}$ is below $7 \%$. However, for the remote sensing of the

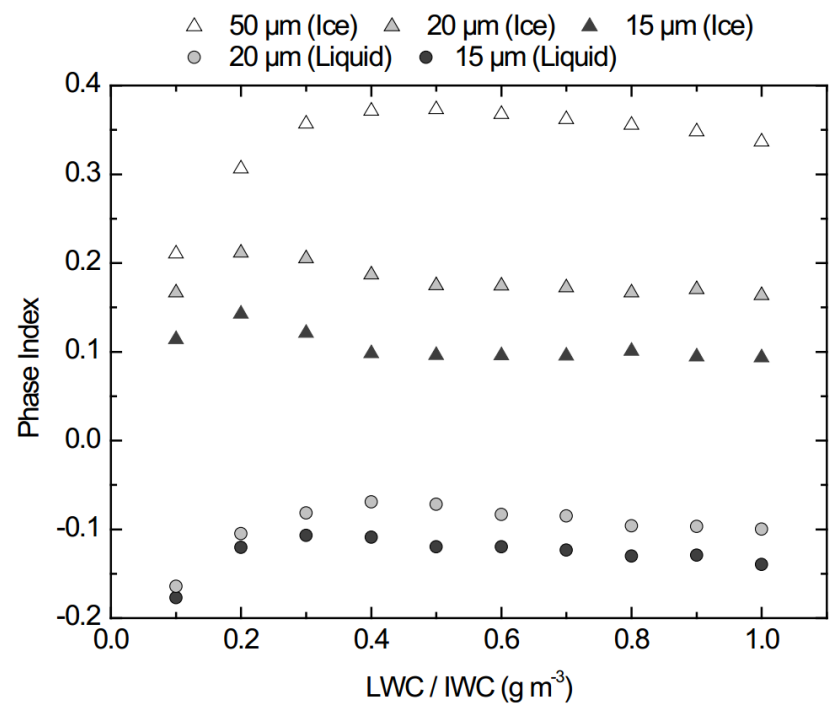

Fig. 3. Phase index dependence on LWC and IWC, respectively, for fixed viewing geometry $\left(\theta_{\mathrm{S}}=50^{\circ}, \varphi_{\mathrm{S}}=0^{\circ}\right)$.

thermodynamic phase the liquid or ice water content is not a critical parameter which needs to be known.

In a second step the phase index $I_{\mathrm{p}}$ is analyzed with respect to the effect of the sensor viewing geometry. $I_{\mathrm{p}}$ is determined for $\varphi_{0}=0^{\circ}, \theta_{0}=30^{\circ}$ and variable sensor azimuth and zenith angles $\left(\varphi_{\mathrm{s}}, \theta_{\mathrm{s}}\right)$ between 0 and $80^{\circ}$ in steps of $10^{\circ}$. For the following example, the effective radius $r_{\text {eff }}$ of the ice and the liquid water cloud is fixed to $15 \mu \mathrm{m}$, while the LWC/IWC is set to $0.7 \mathrm{~g} \mathrm{~m}^{-3}$. Figure 4 shows the relative frequency of the phase indices of both clouds for all points in the model domain from which the reflected radiation of the cloud side are simulated. Overall, there is clear separation between liquid water clouds and ice clouds. Positive values of $I_{\mathrm{p}}$ correspond to ice clouds and negative values indicate a liquid water cloud element. The large range of phase index values indicates a significant impact of the sensor geometry which is also illustrated in Fig. 5a for different $r_{\text {eff }}$ (between 15 and $50 \mu \mathrm{m})$. It presents the derived phase indices for clouds with uniform thermodynamic phase and uniform $r_{\text {eff. Here, sen- }}$ sor and solar azimuth angle are fixed to $0^{\circ}$ which means that the sun is in the back of the observer. For this configuration the sensor zenith angle $\theta_{\mathrm{s}}$ was varied between $20^{\circ}$ and $80^{\circ}$ in $2^{\circ}$ steps. As shown in Fig. 5, the viewing geometry in terms of the scattering angle affects $I_{\mathrm{p}}$ significantly. In this case, an increase of the sensor zenith angle and so the scattering angles (ranging here between 50 and $115^{\circ}$ ) results in an increase of the phase index $I_{\mathrm{p}}$ for ice and liquid water clouds. Note that for the largest sensor zenith angles and large liquid droplets the phase index can exceed values around zero. The geometry effect can be also observed for varying the sensor azimuth angle. In Fig. 5b $I_{\mathrm{p}}$ is shown for ice and liquid water clouds with $r_{\text {eff }}=15 \mu \mathrm{m}$ and a fixed sensor zenith angle of $\theta_{\mathrm{S}}=70^{\circ}$. Again, the sign of the phase index clearly separates 


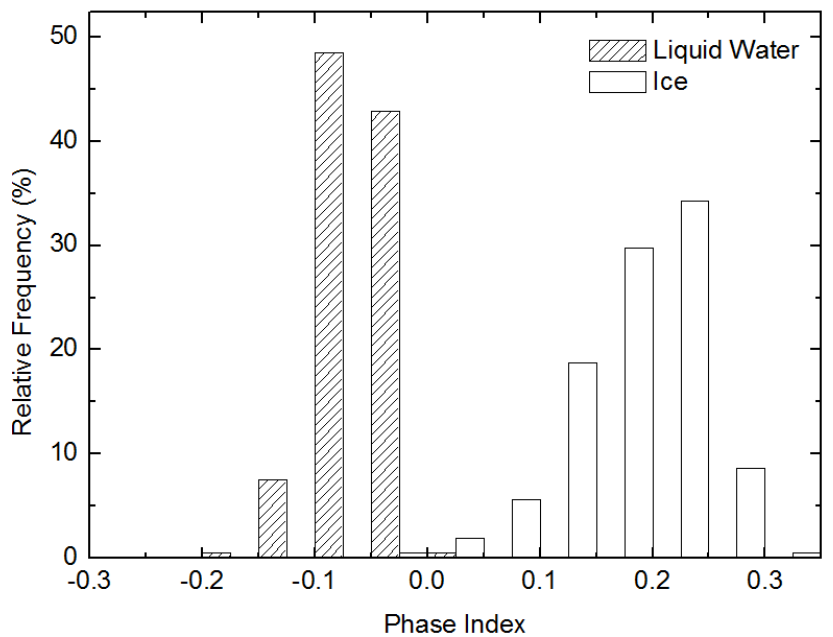

Fig. 4. Relative frequency of phase indices for ice and liquid water clouds $\left(r_{\text {eff }}=15 \mu \mathrm{m}\right)$ for varied sensor viewing geometries based on 600 cases.

the two thermodynamic phases in spite of the varying sensor viewing geometry. In summary, clouds with uniform microphysical parameters can yield a variety of phase indices, depending on the viewing geometry and the cloud particle size. Nevertheless, the thermodynamic phase can clearly be distinguished by the sign of $I_{\mathrm{p}}$.

\subsection{Vertically inhomogeneous clouds}

In a next step, the effective radius and LWC/IWC are varied in vertical. Horizontally the cloud is assumed homogeneous. The vertical extension of the cloud is between 3.8 to $13 \mathrm{~km}$, consisting of ice in the upper part $(7.0-13.0 \mathrm{~km})$ and liquid water in the lower part $(3.8-6.4 \mathrm{~km})$. In between there is a mixed phase layer $(6.4-7.0 \mathrm{~km})$ (see Fig. 6a). Figure $6 \mathrm{~b}$ shows the profile of the single scattering albedo $\tilde{\omega}$ of the individual cloud particles. Due to the wide range of effective radii $(7-44 \mu \mathrm{m}), \tilde{\omega}$ of ice particles varies much stronger $(0.88-0.98$ for $1550 \mathrm{~nm})$ than $\tilde{\omega}$ of the liquid water particles, which is about 0.99 . The profile of the phase index was derived for the side viewing geometry with: $\varphi_{\mathrm{s}}=\varphi_{0}=0^{\circ}$, $\theta_{0}=30^{\circ}$ and a range of $\theta_{\mathrm{S}}$ between 20 and $80^{\circ}$. For a distance to the cloud of about $9 \mathrm{~km}$ (corresponds to $36^{\circ}<\theta_{\mathrm{s}}<66^{\circ}$ ) the profile of the derived phase index is shown in Fig. 6c. It illustrates a clear separation of ice and liquid phase. The differences of the phase index for the ice layer depend strongly on the effective radius. Large effective radii (as at the bottom of the layer) result in a strong absorption due to the decrease of the single scattering albedo. Also the mixed-phase layer can be identified. The variation of the phase index with height for the region of liquid phase is low $(-0.13$ to -0.01$)$ compared to the mixed-phase zone, where the phase index changes rapidly from negative to positive values $(-0.01$ to 0.31 ). When reaching the region with pure ice phase, the
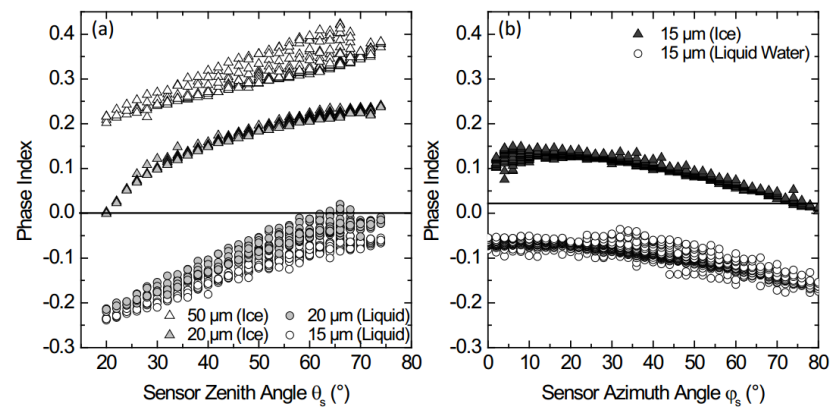

Fig. 5. (a) Phase index derived from water and ice clouds with uniform $r_{\text {eff }}$ and varied sensor zenith angles. (b) Same as (a) but for varied sensor azimuth angle $\left(r_{\mathrm{eff}}=15 \mu \mathrm{m}\right)$.

change of $I_{\mathrm{p}}$ with height is again reduced compared to the mixed-phase layer. However, in the most upper part of the cloud with ice particles of $r_{\text {eff }}<20 \mu \mathrm{m}$, the phase index could be misinterpreted as liquid particles. Here, the single scattering albedo $(\tilde{\omega} \approx 0.98)$ of the small ice particles approaches values that are in the lower range of large liquid particles. In addition to the variable LWC/IWC (open circles in Fig. 6c) also simulations with fixed water content $\left(0.7 \mathrm{~g} \mathrm{~m}^{-3}\right)$ are performed. Their difference is largest in the mixed-phase layer and at the top of the cloud where the variable IWC is much lower than $0.7 \mathrm{~g} \mathrm{~m}^{-3}$. Low extinction due to low IWC at cloud top causes a decrease of the phase index compared to the simulation with fixed water content. A steady decrease of IWC tends to result in a phase index that is similar to a phase index which would be derived for clear sky conditions. There, the phase index depends mainly on the slope of the downward solar spectrum between 1550 and $1700 \mathrm{~nm}$. This negative slope leads to a negative ice index pretending the liquid cloud phase. In the mixed-phase layer of the cloud the impact of the water content gets obvious. Depending on the amount of LWC and IWC either the spectral extinction of the liquid or the ice particles are dominating the phase index. In the lower part of the mixed-phase layer (with LWC $\gg I W C$ ), the phase index is shifted to lower values compared to the simulations with a constant water content, while in the upper part of mixed-phase layer the phase index is shifted to higher values. However, for most of the $I_{\mathrm{p}}$-profile the fixed and the variable water content give similar values. From the above it can be concluded that the sensitivity of the phase index with regard to the water content is negligible above a threshold value which depends on the cloud particle size and the thermodynamic phase.

\section{Measurement example}

Observations of convective clouds passing Leipzig were performed on 25 June 2012. Some of the clouds were precipitating which is an indication of the existence of ice in the cloud. Satellite-based measurements with MODIS 

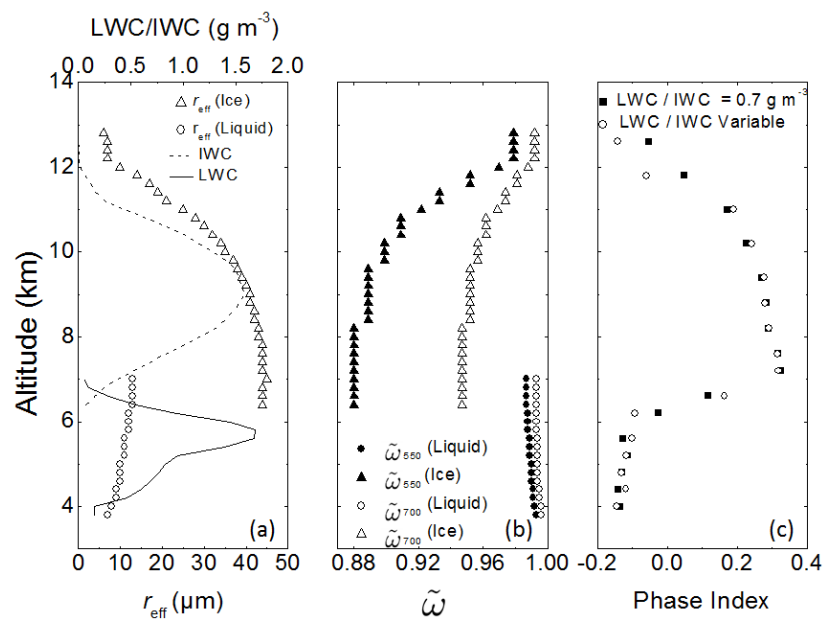

Fig. 6. (a) Vertical profile of $r_{\text {eff }}$ and water contents. (b) Single scattering albedo $\tilde{\omega}$ as derived from Mie-calculation for liquid water and taken from the Baum parametrization for ice particles, and (c) phase index profile.

Table 2. Comparison of phase indices and lidar depolarization slopes.

\begin{tabular}{llll}
\hline & Mixed Phase (1) & Ice Phase (2) & Liquid Phase (4) \\
\hline Lidar $\delta$-slope & 0.01 & 0.02 & 0.005 \\
Measured phase index $I_{\mathrm{p}}$ & 0.02 & 0.33 & -0.17 \\
Simulated phase index $I_{\mathrm{p}}$ & - & $0.31^{\mathrm{a}}$ & $-0.14^{\mathrm{b}}$ \\
\hline
\end{tabular}

${ }^{\mathrm{a}} r_{\text {eff }}=35 \mu \mathrm{m},{ }^{\mathrm{b}} r_{\text {eff }}=10 \mu \mathrm{m}$.

(Moderate-resolution Imaging Spectroradiometer) classified the cloud tops as ice and mixed-phase. The time resolution of the spectrometer measurements was below two seconds, while one lidar profile was sampled within $30 \mathrm{~s}$. Due to the high velocity of the passing clouds (about $20 \mathrm{~m} \mathrm{~s}^{-1}$ ) and their limited horizontal extent, time series at a constant observation angle of the reflected radiance were taken instead of vertical profiles. In many cases individual clouds did not last longer than two minutes.

The data interpretation is supported by photos which also help to sort out shadowed cloud portions which cannot be used for phase discrimination due to the contamination of the spectral slopes of the radiances which are used for the phase discrimination. For fully illuminated, non-shadowed cloud elements, the spectral signature of the reflected signal depends mainly on the spectral signature of the downward solar radiation and its spectral extinction by the observed cloud element. In contrast, for shadowed cloud elements the incident radiation is mostly determined by diffuse radiation. This diffuse radiation is strongly affected by the spectral extinction of the shadowing cloud element but may also be affected by the spectral surface albedo. To identify the illuminated cloud portions all measured spectra were classified with respect to possible contaminations. Figure 7a shows examples
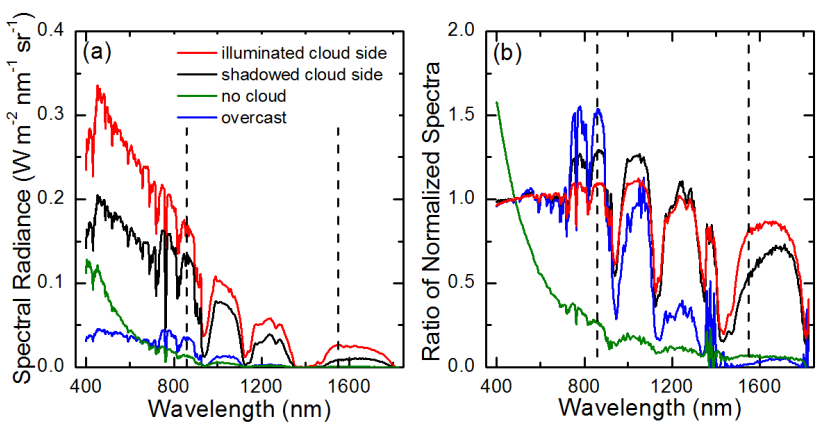

Fig. 7. (a) Spectra of different observing situations: illuminated cloud side, cloud shadow, overcast, and no cloud. (b) Normalized spectral ratios for the cloud scenes from (a) related to a spectrum of illuminated cloud side.

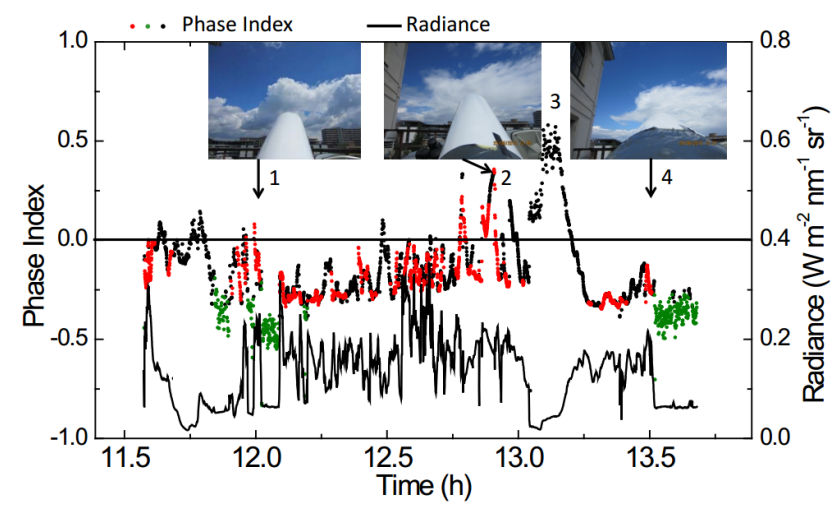

Fig. 8. Time series of spectral radiance at $550 \mathrm{~nm}$ wavelength (black line) and derived phase index (colored circles). The colors indicate the different observing situations: illuminated cloud side (red), cloud shadow/overcast (black), and cloud-free in viewing direction (green).

of measured spectra for different targets: fully illuminated cloud side, shadowed cloud parts, overcast situation, and no cloud. In particular the absolute value of the radiances in the visible spectral range and the slope of the spectrum between 500 and $880 \mathrm{~nm}$ reveals significant differences between the four spectra. In this spectral range the radiance reflected from the illuminated cloud part decreases with increasing wavelength, whereas for spectra with contamination by other clouds the radiance shows an increase at about $730 \mathrm{~nm}$. This feature is an effect of the interaction between clouds and a surface albedo which is affected by vegetation in this particular case. Spectra observed in cloud-free viewing direction show the typical nonlinear decay of the radiance in the VIS caused by Rayleigh scattering. To quantify the spectral differences between reflected radiation from shadowed and illuminated cloud parts, all spectra were normalized to the radiance at $480 \mathrm{~nm}$. Figure $7 \mathrm{~b}$ shows the ratio between the normalized spectra and a normalized spectrum of an illuminated cloud scene. As expected, the most significant difference is 
observed for the cloud-free observation. The overcast situation is characterized by a strong decrease of the ratio in the NIR (ratio $<0.5$ above $1100 \mathrm{~nm}$ ). To distinguish the cloud scenes the radiance ratios at two wavelengths $(857 \mathrm{~nm}$ and $1550 \mathrm{~nm}$, vertical dashed lines in Fig. 7) were calculated. For ratios between 0.8 and 1.2, the cloud scene was defined as illuminated, whereas the cloud-free observations were identified for ratios lower than 0.6. All other spectra were classified as shadowed/overcast cloud scenes.

Figure 8 shows the time series of the measured reflected radiance at $550 \mathrm{~nm}$ indicating the strong variability of the cloud situation (black line in Fig. 8). In Fig. 8, four points of the time series are highlighted. At time step 1, 2 and 4 the radiance is about $0.2 \mathrm{~W} \mathrm{~m}^{-2} \mathrm{~nm}^{-1} \mathrm{sr}^{-1}$, but the corresponding phase index varies between -0.17 (liquid water phase) and +0.33 (ice phase). Furthermore, the mixed-phase can be identified at time step 1 with $I_{\mathrm{p}} \approx 0$. Shortly after time step 1 , even lower phase indices of about -0.6 were observed. In this case the phase index is misleading, because no cloud side reflection is measured. This low phase index results from measurements in cloud-free direction. In contrast, time step 3 shows a phase index of about 0.6 which is much larger than derived for the results of the sensitivity studies. At this time step an overcast situation was observed with a large precipitating convective cloud passing the measurement site, which is also denoted by the low radiance values. However, the high phase index indicates a large ice fraction in the passing cloud which affect the measured spectrum.

In addition to the measured phase indices at time step 1, 2 and 3 , the slope of the depolarization $\delta$ was determined from the lidar measurements. In general, the $\delta$-slope of liquid water clouds is one order of magnitude lower than for ice clouds, which was also found for the time steps 2 and 4 .

Based on the given geometry $\left(\theta_{0}=30^{\circ}, \theta_{\mathrm{s}}=65^{\circ}\right.$, and $\varphi_{0}=$ $\left.\varphi_{\mathrm{S}}=60^{\circ}\right)$, the particle size was varied to derive an phase index close to the measured result (Table 2). For time step 2 an effective radius of $35 \mu \mathrm{m}$ with an ice index of +0.31 (measurement: +0.33) was simulated, while for the liquid water cloud a $r_{\mathrm{eff}}$ of $10 \mu \mathrm{m}$ gave a phase index of -0.14 (measurement: -0.17$)$.

\section{Conclusions}

Ground-based measurements of spectral radiances reflected from convective cloud edges were used to identify the thermodynamic phase of the illuminated cloud portion, which is needed for the retrieval of the effective radius. A thermodynamic phase index was introduced which considers the different spectral slopes for ice and liquid water clouds between $1.55 \mu \mathrm{m}$ and $1.7 \mu \mathrm{m}$. The slope difference results from deviations of the refraction index for ice and liquid water in this spectral range. Sensitivity studies performed with a 3-D radiative transfer model have shown that the phase index is sensitive to the viewing geometry and the effective radius due to the link between single scattering albedo and particle size. Simulations of the cloud edge reflected radiances of homogeneous clouds with constant microphysical properties illustrates that different viewing geometries lead to differences in the phase index which strongly depend on the range of scattering angles. However, in most of the cases the sign of the ice index was a clear indicator of the thermodynamic phase when clouds were composed of pure liquid water or pure ice. For clouds with mixed-phase layers the general statement that a negative slope of the phase index corresponds to liquid water clouds and a positive slope indicates an ice cloud is generally valid. The second sensitivity study investigated the profile of a complex cloud with ice, liquid water and mixedphase layers and variable effective radii and water content. From the profile of the phase index the three layers of liquid water, mixed-phase and ice particles could be identified, which gives confidence that detailed profile measurements of complex clouds can deliver cloud phase formation. The critical mixed-phase layer is indicated by the strong increase of the phase index with height from negative to positive values. Limitations of this method are given by the cloud and viewing geometry. Shadow effects can be omitted by an observing geometry with the sun in the back of the observer. They lead to a misinterpretation of the phase index.

A case study of measured phase indices for passing convective clouds has shown signatures of liquid phase, mixedphase and ice phase which was also found by depolarization measurements of the lidar (slope of depolarization between 0.005 for liquid water and 0.02 for ice phase). From the measured phase index and the given viewing geometry the particle size was estimated with $r_{\text {eff }}$ to be $35 \mu \mathrm{m}$ and $10 \mu \mathrm{m}$ for the ice and liquid water cloud, respectively. It is concluded for this kind of measurements with fast changing cloud scenes that a direct comparison with lidar data is difficult due to its large sampling time. For viewing geometry purposes (sensor distance to the cloud and observed cloud height) the lidar system is essential.

Acknowledgements. This research was funded by the German Research Foundation (DFG, JA 2023/2-1). We thank Sebastian Schmidt and Shi Song from the University of Colorado, Boulder, for their help with the radiative transfer model MCARATS.

Edited by: A. Kokhanovsky

\section{References}

Acarreta, J. R., Stammes, P., and Knap, W. H.: First retrieval of cloud phase from SCIAMACHY spectra around $1.6 \mu \mathrm{m}$, Atmos. Res., 72, 89-105, doi:10.1016/j.atmosres.2004.03.027, 2004.

Anderson, G., Clough, S., Kneizys, F., Chetwynd, J., and Shettle, E.: AFGL Atmospheric Constituent Profiles $(0-120 \mathrm{~km})$, Tech. Rep. AFGL-TR-86-0110, AFGL (OPI), Hanscom AFB, MA 01736, 1986. 
Baum, B., Soulen, P., Strabala, K., King, M., Ackerman, S., Menzel, W., and Yang, P.: Remote sensing of cloud properties using MODIS airborne simulator imagery during SUCCESS. 2. Cloud thermodynamic phase, J. Geophys. Res., 105, 1178111792, 2000.

Baum, B. A., Heymsfield, A. J., Yang, P., and Bedka, S. T.: Bulk scattering properties for the remote sensing of ice clouds. Part I: Microphysical data and models, J. Appl. Meteorol., 44, 1885$1895,2005$.

Baum, B., Yang, P., Nasiri, S., Heidinger, A., Heymsfield, A., and $\mathrm{Li}$, J.: Bulk scattering properties for the remote sensing of ice clouds. Part 3: High resolution spectral models from 100 to $3250 \mathrm{~cm}^{-1}$, J. Appl. Meteorol., 46, 423-434, 2007.

Bierwirth, E., Wendisch, M., Ehrlich, A., Heese, B., Tesche, M., Althausen, D., Schladitz, A., Müller, D., Otto, S., Trautmann, T., Dinter, T., von Hoyningen-Huene, W., and Kahn, R.: Spectral surface albedo over Morocco and its impact on the radiative forcing of Saharan dust, Tellus B, 61, 252-269, 2009.

Bodhaine, B., Wood, N., Dutton, E., and Slusser, J.: On Rayleigh oprical depth calculations, J. Atmos. Ocean. Tech., 16, 18541861, 1999.

Chang, F.-L. and Li, Z.: Estimating the vertical variation of cloud droplet effective radius using multispectral near-infrared satellite measurements, J. Geophys. Res., 107, 7.1-7.12, doi:10.1029/2001JD000766, 2002.

Chang, F.-L. and Li, Z.: Retrieving vertical profiles of watercloud droplet effective radius: Algorithm modification and preliminary application, J. Geophys. Res., 108, 4763, doi:10.1029/2003JD003906, 2003.

Ehrlich, A.: The Impact of Ice Crystals on Radiative Forcing and Remote Sensing of Arctic Boundary-Layer Mixed-Phase Clouds, Ph.D. thesis, Johannes Gutenberg University Mainz, Germany, 2009.

Ehrlich, A., Wendisch, M., Bierwirth, E., Herber, A., and Schwarzenböck, A.: Ice crystal shape effects on solar radiative properties of Arctic mixed-phase clouds - Dependence on microphysical properties, Atmos. Res., 88, 266-276, 2008.

Eichler, H., Ehrlich, A., Wendisch, M., Mioche, G., Gayet, J.F., Wirth, M., Emde, C., and Minikin, A.: Influence of ice crystal shape on retrieval of cirrus optical thickness and effective radius: A case study, J. Geophys. Res., 114, D19203, doi:10.1029/2009JD012215, 2009.

Freud, E., Rosenfeld, D., Andreae, M. O., Costa, A. A., and Artaxo, P.: Robust relations between $\mathrm{CCN}$ and the vertical evolution of cloud drop size distribution in deep convective clouds, Atmos. Chem. Phys., 8, 1661-1675, doi:10.5194/acp-8-1661-2008, 2008.

Hu, Y.-X., Winker, D., Yang, P., Baum, B., Poole, L., and Vann, L.: Identification of cloud phase from PICASSO-CENA lidar depolarization: a multiple scattering sensitivity study, J. Quant. Spectrosc. Ra., 70, 569-579, 2001.

IPCC: Climate Change 2007: The Physical Science Basis, Cambridge University Press, UK, 2007.

Iwabuchi, H.: Efficient Monte Carlo methods for radiative transfer modeling, J. Atmos. Sci., 63, 2324-2339, 2006.

Iwabuchi, H. and Kobayashi, H.: Modeling of radiative transfer in cloudy atmospheres and plant canopies using Monte Carlo methods, Tech. rep., FRCGC, No. 8, 199 pp., 2008.
Jäkel, E., Wendisch, M., Kniffka, A., and Trautmann, T.: Airborne system for fast measurements of upwelling and downwelling spectral actinic flux densities, Appl. Optics, 44, 434-444, 2005.

Khain, A., BenMoshe, N., and Pokrovsky, A.: Factors determining the impact of aerosols on surface precipitation from clouds: Attempt of classification, J. Atmos. Sci., 65, 1721-1748, 2008.

Knap, W., Stammes, P., and Koelemeijer, R.: Cloud thermodynamic-phase determination from near-infrared spectra of reflected sunlight, J. Atmos. Sci., 59, 83-96, 2002.

Kokhanovsky, A. and Rozanov, V. V.: Droplet vertical sizing in warm clouds using passive optical measurements from a satellite, Atmos. Meas. Tech., 5, 517-528, doi:10.5194/amt-5-517-2012, 2012.

Koren, I., Kaufman, Y., Remer, L., and Martins, J.: Measurement of the Effect of Amazon Smoke on Inhibition of Cloud Formation, Science, 303, 1342-1345, 2004.

Lohmann, U. and Feichter, J.: Global indirect aerosol effects: a review, Atmos. Chem. Phys., 5, 715-737, doi:10.5194/acp-5-7152005, 2005.

Martins, J. V., Marshak, A., Remer, L. A., Rosenfeld, D., Kaufman, Y. J., Fernandez-Borda, R., Koren, I., Correia, A. L., Zubko, V., and Artaxo, P.: Remote sensing the vertical profile of cloud droplet effective radius, thermodynamic phase, and temperature, Atmos. Chem. Phys., 11, 9485-9501, doi:10.5194/acp-11-94852011, 2011.

Pierluissi, J. and Peng, G.-S.: New molecular transmission band models for LOWTRAN, Opt. Eng., 24, 541-547, 1985.

Pilewskie, P. and Twomey, S.: Discrimination of ice from water in clouds by optical remote sensing, Atmos. Res., 21, 113-122, 1987.

Platnick, S.: Vertical photon transport in cloud remote sensing problems, J. Geophys. Res., 105, 22919-22935, doi:10.1029/2000JD900333, 2000.

Ricchiazzi, P. and Gautier, C.: Investigation of the effect of surface heterogeneity and topography on the radiation environment of Palmer Station, Antarctica, with a hybrid 3-D radiative transfer model, J. Geophys. Res., 103, 6161-6178, 1998.

Rosenfeld, D.: Suppression of rain and snow by urban and industrial air pollution, Science, 287, 1793-1796, 2000.

Rosenfeld, D. and Lensky, I.: Satellite-based insights into precipitation formation processes in continental and maritime convective clouds, B. Am. Meteorol. Soc., 79, 2457-2476, 1998.

Sassen, K.: The polarization lidar technique for cloud research: a review and current assessment, B. Am. Meteorol. Soc., 72, 1848 1866, 1991.

Scotland, R. M., Sassen, K., and Stone, R.: Observations by lidar of linear depolarizations of hydrometeors, J. Appl. Meteorol., 10, 1011-1017, 1971.

Strabala, K. and Ackerman, S.: Cloud properties from $8-12 \mu \mathrm{m}$ data, J. Appl. Meteorol., 33, 212-229, 1994.

Turner, D. D., Ackerman, S. A., Baum, B. A., Revercomb, H. E., and Yang, P.: Cloud phase determination using ground-based AERI observations at SHEBA, J. Appl. Meteorol., 42, 701-715, 2003.

Wang, X., Liou, K. N., Ou, Steve S. C., Mace, G. G. and Deng, M.: Remote sensing of cirrus cloud vertical size profile using MODIS data, J. Geophys. Res., 114, D09205, doi:10.1029/2008JD011327, 2009. 
Wendisch, M., Müller, D., Schell, D., and Heintzenberg, J.: An airborne spectral albedometer with active horizontal stabilization, J. Atmos. Ocean. Tech., 18, 1856-1866, 2001.

Zhang, Z., Platnick, S., Yang, P., Heidinger, A. K., and Comstock, J. M.: Effects of ice particle size vertical inhomogeneity on the passive remote sensing of ice clouds, J. Geophys. Res., 115, D17203, doi:10.1029/2010JD013835, 2010.
Zinner, T., Marshak, A., Lang, S., Martins, J. V., and Mayer, B.: Remote sensing of cloud sides of deep convection: towards a three-dimensional retrieval of cloud particle size profiles, Atmos. Chem. Phys., 8, 4741-4757, doi:10.5194/acp-8-4741-2008, 2008. 